\title{
BMJ Open Pharmacological interventions to improve sleep in hospitalised adults: a systematic review
}

\author{
Salmaan Kanji, ${ }^{1,2}$ Alexandru Mera, ${ }^{3}$ Brian Hutton, ${ }^{2,4}$ Lisa Burry, ${ }^{5}$ Erin Rosenberg, ${ }^{6}$ \\ Erika MacDonald, ${ }^{2,7}$ Vanessa Luks ${ }^{8}$
}

To cite: Kanji S, Mera A, Hutton B, et al. Pharmacological interventions to improve sleep in hospitalised adults: a systematic review. BMJ Open 2016;6: 012108. doi:10.1136/bmjopen-2016012108

- Prepublication history and additional material is available. To view please visit the journal (http://dx.doi.org/ 10.1136/bmjopen-2016012108).

SK and AM contributed equally to this work and would like to be recognised as coprimary authors.

Received 7 April 2016 Revised 15 June 2016 Accepted 4 July 2016

CrossMark

For numbered affiliations see end of article.

Correspondence to

Dr Salmaan Kanji;

skanji@toh.on.ca

\section{ABSTRACT}

Objectives: Patients often suffer from disturbed sleep in hospital. Poor-quality sleep in hospitalised patients has been associated with significant morbidity and pharmacological sleep aids are often prescribed. The objective of this systematic review is to evaluate the comparative efficacy and safety of pharmacological interventions used for sleep in hospitalised patients.

Setting/participants: We searched MEDLINE, Embase, the Cochrane database and grey literature for prospective studies that evaluated sleep in hospitalised adults after a pharmacological intervention.

Primary and secondary outcome measures: Two reviewers assessed studies for inclusion and extracted data for efficacy outcomes, including sleep efficiency, sleep latency, sleep fragmentation and objectively measured sleep stage distribution. Risk of bias was assessed and meta-analyses were planned contingent upon homogeneity of the included studies.

Results: After screening 1920 citations, 15 studies involving 861 patients were included. Medications studied included benzodiazepines, nonbenzodiazepine sedatives, melatonin, propofol and dexmedetomidine. Five studies were deemed to be of high quality. Heterogeneity and variable outcome reporting precluded meta-analysis in most cases. No consistent trends with respect to sleep efficiency, quality or interruptions were observed identifying a drug or drug class as superior to another or no treatment. Benzodiazepines appeared to be better than no treatment with respect to sleep latency, but this was not consistently demonstrated across all studies. Sleep stage distribution shows that sleep in hospital is dominated by stages N1 and N2.

Conclusions: There is insufficient evidence to suggest that pharmacotherapy improves the quality or quantity of sleep in hospitalised patients suffering from poor sleep. No drug class or specific drug was identified as superior even when compared to placebo or no treatment. Although 15 studies were included, the quality of evidence was limited by their quality and size. Larger, better-designed trials in hospitalised adults are needed.

\section{BACKGROUND}

Sleep disturbances, including difficulty initiating sleep, fragmented and non-restorative

\section{Strengths and limitations of this study}

- The use of a validated systematic search strategy of multiple databases to identify relevant studies.

- Article screening and data extracted independently by two investigators.

- The research team consisted of physicians, pharmacists, an epidemiologist/methodologist, a sleep expert and pharmacotherapy specialists.

- The studies included were typically small, outcomes were often measured subjectively and most studies did not have a standard approach to the evaluation of safety.

- Not all drugs currently being used in clinical settings were identified in clinical trials and comparative trials were identified without placebo controls for many drugs.

sleep, occur in up to $50 \%$ of acutely ill hospitalised patients. ${ }^{1}$ Hospitalised patients routinely suffer from sleep disorders attributed to pathophysiological and environmental factors. $^{2}{ }^{3}$ In a study of 280 hospitalised elderly patients, $21 \%$ reported new-onset insomnia, $38 \%$ reported moderate or severe insomnia and $38 \%$ reported sleep disturbances during admission. ${ }^{4}$ The transient nature of acute illness coupled with the displacement and hospitalisation of patients represent unique features that differentiate insomnia associated with acute illness and hospitalisation from insomnia in otherwise healthy people. Sleep deprivation has been associated with poor wound healing, memory disorders, delayed ventilator weaning, delirium and mortality. ${ }^{5-8}$ Given the frequency of sleep disorders experienced by hospitalised patients and their associated outcomes, interventions to improve sleep are frequently attempted. ${ }^{1} 910$ Non-pharmacological interventions such as relaxation techniques and regulation of light/noise exposure are often tried to counter newonset sleep disorders related to the environment. ${ }^{1}{ }^{10}$ However, a systematic review of 
non-pharmacological interventions for sleep compared to no intervention (in hospitalised but non-critically ill patients) did not confirm a positive effect, citing a lack of high-quality studies. ${ }^{11}$ While environmental factors (related to light, noise and activity) are common in hospital and particularly in the intensive care unit (ICU), limiting the potential impact of these on sleep quality is not always easily accomplished. Accordingly, sedative and hypnotic pharmacotherapy is used in up to $88 \%$ of hospitalised patients. ${ }^{12}$

Currently, there are no evidence-based recommendations or guidelines to direct the choice of hypnotic drugs in the hospital setting, yet there are a plethora of agents that are used. The objective of this systematic review was to identify, synthesise and summarise the existing evidence on the efficacy and safety of pharmacological interventions used to improve sleep in hospitalised adults.

\section{METHODS}

A protocol was developed prior to initiation of the review and was followed throughout the review process. The protocol is available from the authors electronically upon request.

\section{Selection of studies}

The Population, Intervention, Comparator, Outcome and Study design framework was used to formulate the research question for this systematic review. ${ }^{13}$ The population of interest was hospitalised adults in an attempt to identify acutely ill patients. Studies of patients in any acute care ward of a hospital (including medical, surgical and critical care units) were sought and non-hospitalised patients (ie, healthy volunteers, outpatients and longterm care facility residents) were excluded. We also excluded studies conducted solely in patients diagnosed with a primary psychiatric illness or sleep apnoea as these patient populations exhibit different baseline sleep patterns irrespective of environment. ${ }^{1}$ Interventions of interest for this systematic review included any pharmacological intervention for sleep used alone or in combination. This included benzodiazepines, antidepressants, antihistamines, imidazopyridines (eg, zolpidem), pyrazolopyrimidines (eg, zaleplon), cyclopyrrolones (eg, zopiclone), anticonvulsants (pregabalin/gabapentin), barbiturates, $\alpha$-2-adrenergic agonists, antipsychotics, melatonin or melatonin receptor agonists and natural health products. Studies involving drugs that are no longer commercially available in any country were excluded. No restrictions on dose for any agent were employed. Comparators of interest included placebo, no treatment or any other intervention.

Efficacy outcomes of interest were sleep efficiency, sleep latency, sleep interruptions and sleep stage distribution measured by any means, including polysomnography, bispectral index monitoring (BIS), actigraphy, sleep questionnaires for patient self-reporting or direct observation by a third party. However, studies solely relying on patient self-reporting for sleep outcomes of interest were excluded as subjective reports are poorly correlated with objective measures and biased by systematic over-reporting. ${ }^{14}{ }^{15}$ Sleep efficiency is defined as hours spent asleep divided by period of observation (in hours) by study investigators, while sleep latency represents the duration of time taken to fall asleep. Sleep fragmentation is defined as the number of arousals or awakenings in a designated sleep period. Sleep stage distribution refers to the proportion of time a patient spends in each sleep stage in a given period of observation. Secondary clinical outcomes included length of stay, duration of mechanical ventilation, presence of delirium, occurrence of infection, cognitive function, pain, glucose control, hypertension, myocardial infarction and mortality. Safety outcomes of interest included overall occurrence of adverse events, serious adverse events, including those deemed life threatening, and withdrawal or discontinuation of therapy due to adverse events. Study design comprised randomised controlled trials of pharmacological interventions for sleep. Additionally, comparative cohort studies and prospective case series were included only if sleep was evaluated using polysomnography. The language of publication was restricted to English or French. ${ }^{16}$

\section{Literature search strategy}

Relevant studies were identified using a sequential search approach. Initially, an informal scoping exercise was conducted to identify potentially relevant trials in Ovid MEDLINE with key words such as 'sleep', 'insomnia', 'drug therapy' and 'in-patient'. Relevant studies identified in the scoping exercise were provided to an information specialist to develop and validate a search strategy for Ovid MEDLINE, Embase and Cochrane from inception to 21 March 2016. The search strategy was peer-reviewed using PRESS (Peer Review of Electronic Search Strategies) by a second information specialist and validated using the studies identified in the scoping exercise. ${ }^{17}$ The detailed search strategy is provided in online supplementary appendix 1. In addition, the reference lists of included studies and reviews were screened.

Grey literature was searched via conference proceedings and abstracts (Canadian Sleep Society, American Academy of Sleep Medicine and European Sleep Research Society) for the last 5 years, as well as clinical trial and systematic review registries, and Google Scholar. ${ }^{18}$

Titles and abstracts were independently reviewed for eligibility by two investigators (AM and SK). Full-text articles identified as potentially eligible at first-level screening were obtained and reviewed for eligibility independently by the same two reviewers. Discrepancies were resolved by discussion and consensus. The process of study selection was documented using a PRISMA flow diagram. ${ }^{19}$

\section{Data extraction and risk of bias assessment}

Study level data were extracted using a predesigned and piloted data collection form by one author (AM) and 
checked by a second (SK). Means and SDs were estimated using established methods when only medians and ranges were available from an included study. ${ }^{20} 21$ In addition to the clinical and safety outcomes, extracted study data included the following: study authors, year and journal of publication, country of study performance, funding source, group sample sizes, study inclusion criteria, age distribution, gender distribution, patient setting in hospital and relevant concomitant medication use. Risk of bias was assessed using the Cochrane Risk of Bias tool ${ }^{22}$ for randomised controlled trials and the Newcastle-Ottawa Scale ${ }^{23}$ for cohort studies. Risk of bias was not assessed for prospective case series, as the risk of bias is inherently high.

\section{Synthesis of available studies}

Clinical and methodological heterogeneity of the included studies was carefully considered to determine whether meta-analysis would be conducted. Clinical heterogeneity was assessed by comparing study populations, interventions and outcome measurements between studies. ${ }^{24}$ Methodological heterogeneity was assessed by comparing methods for intervention assignment, allocation concealment and blinding, as well as the extent of and reasons for loss to follow-up. If heterogeneity was not identified between trials and reporting of clinical outcomes was amenable to meta-analysis, a randomeffects model was employed to calculate pooled risk ratios for dichotomous outcomes (ie, adverse events) and pooled mean differences for continuous outcomes (ie, sleep efficiency) using RevMan V.5.3 (the Nordic Cochrane Center, the Cochrane Collaboration, Copenhagen, Denmark). ${ }^{25}{ }^{26}$ Studies were to be pooled by drug and by drug class and compared with active controls or placebo separately depending on the availability of data. In the event that 10 or more studies were pooled for any outcome, funnel plots would be created and visually inspected to assess for publication bias. ${ }^{22}$

The PRISMA statement and its 27-item checklist were used in developing the summary of findings for this review. A completed PRISMA checklist is provided in online supplementary appendix $2{ }^{19}$

\section{RESULTS}

\section{Characteristics of included trials}

Our search strategy identified a total of 1920 citations, from which 11 randomised controlled trials, 2 prospective cohort studies and 2 case series, totalling 861 patients, met criteria for this review (figure 1). Twenty-eight studies were excluded because the only method of sleep assessment was via patient self-report. Eight studies were conducted in the ICU and included patients requiring mechanical ventilation. Seven studies were conducted in acute care hospital wards and included medical and surgical patients suffering from inadequate sleep. Six studies measured sleep using polysomnography, two used BIS and the remaining measured sleep using either third-party observation or a combination of third-party observation and patient selfreport (tables 1 and 2). Medications studied included benzodiazepines, melatonin, propofol, zolpidem, zopiclone and dexmedetomidine. In most studies, the intervention was administered in the evening and sleep was evaluated over night; however, two of the ICU-based studies evaluated sleep while on continuous infusions of sedatives. Study durations ranged from 24 hours to 14 days. Seven of the randomised controlled trials had a high risk of bias in at least one criteria, which affected their overall quality (figure 2). The prospective cohort studies included were of high quality (figure 3 ).
Figure 1 Process of study identification and selection. *Prospective cohort studies not using polysomnography to measure sleep. RCT, randomised controlled trial.

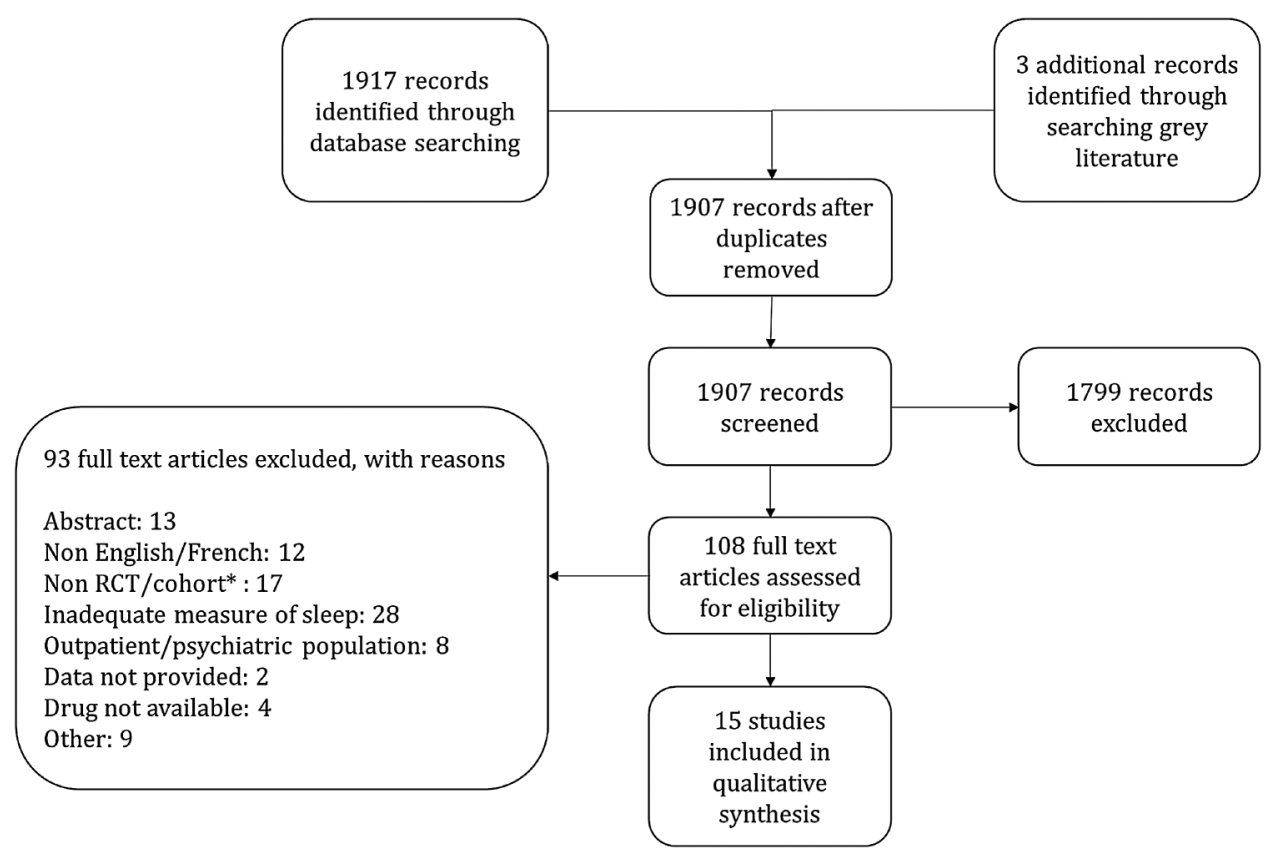




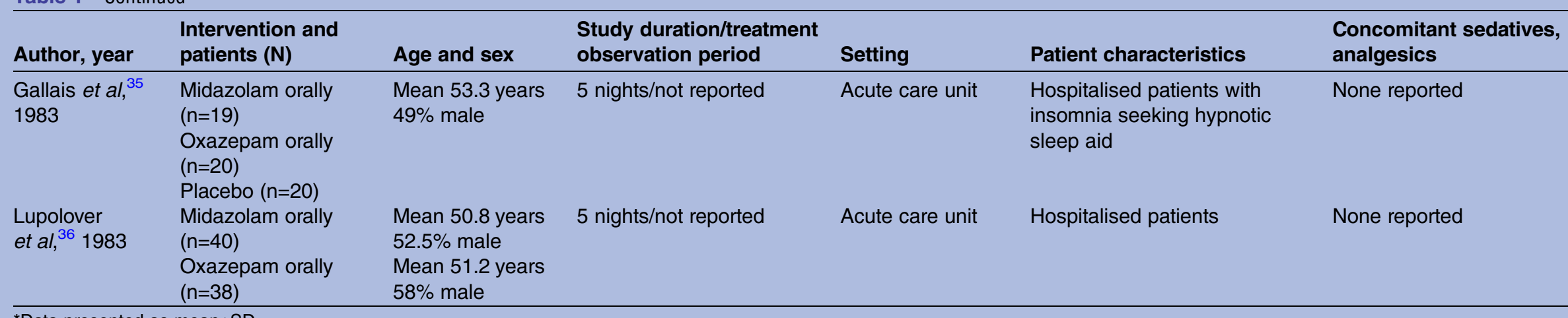

${ }^{*}$ Data presented as mean $\pm \mathrm{SD}$.

†Data presented as median (IQR).

†Data presented as mean $(95 \% \mathrm{Cl})$.

§Data presented as median (range).

BIS, bispectral index; GCS, Glasgow coma scale; ICU, intensive care unit; iv, intravenous.

Table 2 Study characteristics: cohort studies and case series

\begin{tabular}{|c|c|c|c|c|c|c|}
\hline $\begin{array}{l}\text { Author, year } \\
\text { (design) }\end{array}$ & Intervention and patients (N) & Age and sex & $\begin{array}{l}\text { Study duration/ } \\
\text { treatment } \\
\text { observation period }\end{array}$ & Setting & Patient characteristics & $\begin{array}{l}\text { Concomitant } \\
\text { sedatives, } \\
\text { analgesics }\end{array}$ \\
\hline $\begin{array}{l}\text { Alexopoulou et al, } \\
2014 \text { (cohort with } \\
\text { crossover) }\end{array}$ & $\begin{array}{l}\text { Dexmedetomidine iv infusion } \\
(n=13) \\
\text { No treatment }(n=13)\end{array}$ & $\begin{array}{l}62(55-69) \text { years* } \\
77 \% \text { male }\end{array}$ & 57 hours $/ 9$ hours & $\begin{array}{l}\text { Critical } \\
\text { care unit }\end{array}$ & $\begin{array}{l}\text { Mechanically ventilated ICU } \\
\text { patients }\end{array}$ & None reported \\
\hline $\begin{array}{l}\text { Kim et al, }{ }^{39} 2014 \\
\text { (case series) }\end{array}$ & Midazolam iv infusion $(n=5)$ & $\begin{array}{l}67(40.5-72) \text { years }^{*} \\
80 \% \text { male }\end{array}$ & 24 hours/24 hours & $\begin{array}{l}\text { Critical } \\
\text { care unit }\end{array}$ & $\begin{array}{l}\text { Mechanically ventilated ICU } \\
\text { patients requiring continuous } \\
\text { sedation }\end{array}$ & None reported \\
\hline $\begin{array}{l}\text { Oto et al, }{ }^{40} 2012 \\
\text { (case series) }\end{array}$ & $\begin{array}{l}\text { Dexmedetomidine iv infusion } \\
(n=10)\end{array}$ & $\begin{array}{l}68 \pm 9 \text { years } \dagger \\
30 \% \text { male }\end{array}$ & 24 hours/9 hours & $\begin{array}{l}\text { Critical } \\
\text { care unit }\end{array}$ & $\begin{array}{l}\text { Mechanically ventilated ICU } \\
\text { patients requiring iv sedation }\end{array}$ & None reported \\
\hline $\begin{array}{l}\text { Hardin et al, }{ }^{33} 2006 \\
\text { (cohort) }\end{array}$ & $\begin{array}{l}\text { Lorazepam iv (intermittent } \\
\text { sedation) }(n=6) \\
\text { Lorazepam iv (continuous } \\
\text { sedation) }(n=6) \\
\text { Lorazepam iv (continuous } \\
\text { sedation with neuromuscular } \\
\text { blockade) }(n=6)\end{array}$ & $\begin{array}{l}58.5 \pm 9.8 \text { years } \dagger \\
17 \% \text { male } \\
43.2 \pm 13 \text { years } \dagger \\
33 \% \text { male } \\
61.3 \pm 8.8 \text { years } \dagger \\
83 \% \text { male }\end{array}$ & 24 hours/24 hours & $\begin{array}{l}\text { Critical } \\
\text { care unit }\end{array}$ & $\begin{array}{l}\text { Mechanically ventilated ICU } \\
\text { patients }\end{array}$ & None reported \\
\hline
\end{tabular}




\begin{tabular}{|l|l|l|l|l|l|l|}
\hline $\begin{array}{l}\text { Study } \\
\text { (Author, year } \\
\text { of publication) }\end{array}$ & $\begin{array}{l}\text { Random } \\
\text { sequence } \\
\text { generation }\end{array}$ & $\begin{array}{l}\text { Allocation } \\
\text { concealment }\end{array}$ & $\begin{array}{l}\text { Blinding of } \\
\text { participants } \\
\text { and } \\
\text { personnel }\end{array}$ & $\begin{array}{l}\text { Blinding of } \\
\text { outcome } \\
\text { assessment }\end{array}$ & $\begin{array}{l}\text { Incomplete } \\
\text { outcome } \\
\text { data }\end{array}$ & $\begin{array}{l}\text { Selective } \\
\text { reporting }\end{array}$ \\
\hline $\begin{array}{l}\text { Engelman et } \\
\text { al., 2014 }\end{array}$ & Moderate & Moderate & Moderate & Moderate & Low & Low \\
\hline $\begin{array}{l}\text { Kondili et al., } \\
\mathbf{2 0 1 2}\end{array}$ & Moderate & High & High & Low & Low & Low \\
\hline $\begin{array}{l}\text { Oto et al., } \\
\mathbf{2 0 1 1}\end{array}$ & Low & Moderate & High & High & Low & Low \\
\hline $\begin{array}{l}\text { Bourne et al., } \\
\mathbf{2 0 1 1}\end{array}$ & Low & Moderate & Low & Moderate & Low & Low \\
\hline $\begin{array}{l}\text { lbrahim et al., } \\
\mathbf{2 0 0 6}\end{array}$ & Low & Low & Low & High & Moderate & Moderate \\
\hline $\begin{array}{l}\text { Li Pi Shan et al., } \\
\mathbf{2 0 0 4}\end{array}$ & Low & Moderate & Moderate & Moderate & High & Low \\
\hline $\begin{array}{l}\text { Morgan et al., } \\
\mathbf{2 0 0 4}\end{array}$ & Low & Low & Moderate & Moderate & Low & Low \\
\hline $\begin{array}{l}\text { Feldmeier and } \\
\text { Kapp, 1986 }\end{array}$ & Moderate & Low & Moderate & Low & Low & Low \\
\hline $\begin{array}{l}\text { Goetzke et al., } \\
\mathbf{1 9 8 3}\end{array}$ & Low & Moderate & Moderate & Moderate & High & Low \\
\hline $\begin{array}{l}\text { Gallais et al., } \\
\mathbf{1 9 8 3}\end{array}$ & Low & Low & Low & Moderate & High & Low \\
\hline $\begin{array}{l}\text { Lupolover et } \\
\text { al., } \mathbf{1 9 8 3}\end{array}$ & Moderate & Moderate & Moderate & Moderate & High & Low \\
\hline
\end{tabular}

Figure 2 Risk of bias for randomised controlled trials using the Cochrane risk of bias tool. Risk of bias assessed as low, moderate or high for each category.

\section{Sleep efficiency}

Sleep efficiency was evaluated in seven studies (five randomised controlled trials ${ }^{27-31}$ and two cohort studies $^{32}{ }^{33}$ ), of which two ${ }^{31} 32$ identified statistically significant differences (table 3, figure 4). Alexopoulou et $a \vec{l}^{2}$ evaluated sleep with polysomnography in 13 of 16 enrolled patients over the course of three nights in a prospective crossover cohort study. On nights 1 and 3, patients received no treatment, while on night 2 , they received dexmedetomidine. Polysomnography was not consistently viable, and only 10 patients contributed to the reported analysis, which showed that sleep efficiency was improved with dexmedetomidine $(77.9 \%$ (SD 65.6$80.2 \%)$ ) when compared to no treatment $(15.8 \%$ (6.4$51.6 \%), \mathrm{p}=0.002)$. In a study by Oto $e t a l^{31}$ two different dosing strategies for midazolam were compared in a randomised controlled trial of 22 critically ill patients. Both groups received sedation with a continuous infusion of midazolam, but one group had their infusions interrupted during the day. Sleep efficiency as measured by polysomnography was statistically significantly improved $(\mathrm{p}=0.047)$ in the group that did not have their infusion interrupted during the day $(97 \%(91.1-97.8 \%)$ vs $81 \%$ $(73.3-91.1 \%))$. In a similar trial of 18 patients by Hardin $e t a l,{ }^{33}$ critically ill adults were administered lorazepam via continuous infusion or an interrupted dosing strategy; unlike in the previous study, differences between groups were not statistically significant (table 3, figure 4).

Two randomised controlled trials (Bourne et $a l^{27}$ and Ibrahim et $a l^{28}$ ) that compared melatonin with placebo failed to show a statistically significant difference in sleep efficiency as measured by BIS or nurse observation. Similarly, two trials by Engelmann et a $\vec{l}^{30}$ and Kondili et $a l^{29}$ comparing propofol to flunitrazepam and no treatment did not show statistically significant differences with respect to sleep efficiency as measured by BIS and polysomnography, respectively. Only the two placebocontrolled melatonin trials (total of 56 patients) were amenable to pooling of this outcome that revealed a
Figure 3 Risk of bias for prospective cohort studies (NewCastle-Ottawa Scale).

\begin{tabular}{|l|c|c|c|}
\hline $\begin{array}{l}\text { Study } \\
\text { (Author, year of } \\
\text { publication) }\end{array}$ & $\begin{array}{l}\text { Selection of Studies } \\
\text { (max of } \mathbf{4}^{*} \text { ) }\end{array}$ & $\begin{array}{l}\text { Comparability of } \\
\text { Groups } \\
\text { (max of 2 }\end{array}$ & $\begin{array}{l}\text { Ascertainment of } \\
\text { exposure/outcome } \\
\text { (max of 3 *) }\end{array}$ \\
\hline Alexopolou et al., 2014 & $* * * *$ & $* *$ & $* *$ \\
\hline Hardin et al., 2006 & $* * * *$ & $* *$ & $* *$ \\
\hline
\end{tabular}


Table 3 Summary of study findings, by outcome

\section{Author, year Treatment group}

Mechanically ventilated patients in an ICU

Alexopoulou Dexmedetomidine iv infusion

et $\mathrm{al}^{32} 2014 \quad$ (0.6 (0.4-0.7) $\left.\mu \mathrm{g} / \mathrm{kg} / \mathrm{hour}\right)^{*}$

$(n=16$ enrolled, 13 analysed)

No treatment crossove

$(n=16$ enrolled, 10 analysed)

Kim et $a l,{ }^{39}$

2014
Midazolam iv $0.02(0.015-0.04)$ $\mathrm{mg} / \mathrm{kg} / \mathrm{hour}^{*}$ ( $\mathrm{n}=9$ enrolled, 5 analysed)
Polysomnography

Polysomnography
Polysomnography (no treatment on day 1 , dexmedetomidine on day 2)

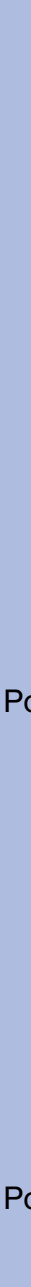

Outcome by

treatment group

Sleep efficiency ${ }^{\star}$

$77.9 \%(65.6-80.2 \%)$

Sleep stage distribution

$(\%)^{\star}$

Stage $\mathrm{I}=16.1(6.2-21.3)$

Stage II=78.7 (69.2-92.5)

Stage III=0.0 (0-0)

REM=0.0 (0-0.4)

Sleep fragmentations ${ }^{\star}$

2.2 (1.6-4.5) arousals/

hour

Sleep efficiency ${ }^{*}$

$15.8 \%(6.4-51.6 \%)$

Sleep stage distribution

$(\%)^{\star}$

Stage I=56.2 (24.7-79.3)

Stage II=39.2 (20.7-66.4)

Stage III=0.0 (0-0)

REM $=0.0(0-0.4)$

Sleep fragmentation

7.1 (6.1-13.4) arousals/

hour

Sleep efficiency ${ }^{*}$

$34.3 \%$ (8-59.7)

Sleep stage distribution

$(\min )^{\star}$

Stage $\mathrm{I}=82.0$

(60.5-372.5)

Stage $\|=88.0$

(19.0-621.0)

Stage III=0.0 (0-0)

$\mathrm{REM}=10.0$ (6.0-50.5)

Sleep fragmentation ${ }^{*}$

16.1 (7.6-28.6) arousals/

hour

Sleep efficiency ${ }^{*}$

$52.3 \%(47-89.7)$

Sleep fragmentation ${ }^{*}$

9.3 (3-19.5) arousals/hou
Measure of effect

Sleep efficiency

$\mathrm{p}=0.002$ between first and second nights;

$p=0.001$ between second and third nights

Sleep fragmentation

$\mathrm{p}=0.0023$

Sleep stagedistribution

Stage I: $p=0.006$

Stage II: $p=0.006$

Stage III: $p=0.180$

REM: $p=0.173$

Correlation between dose of midazolam and total sleep time $(r=0.975, p=0.005)$

Correlation between midazolam dose and stage

II $(r=0.975, p=0.005)$

None reported 


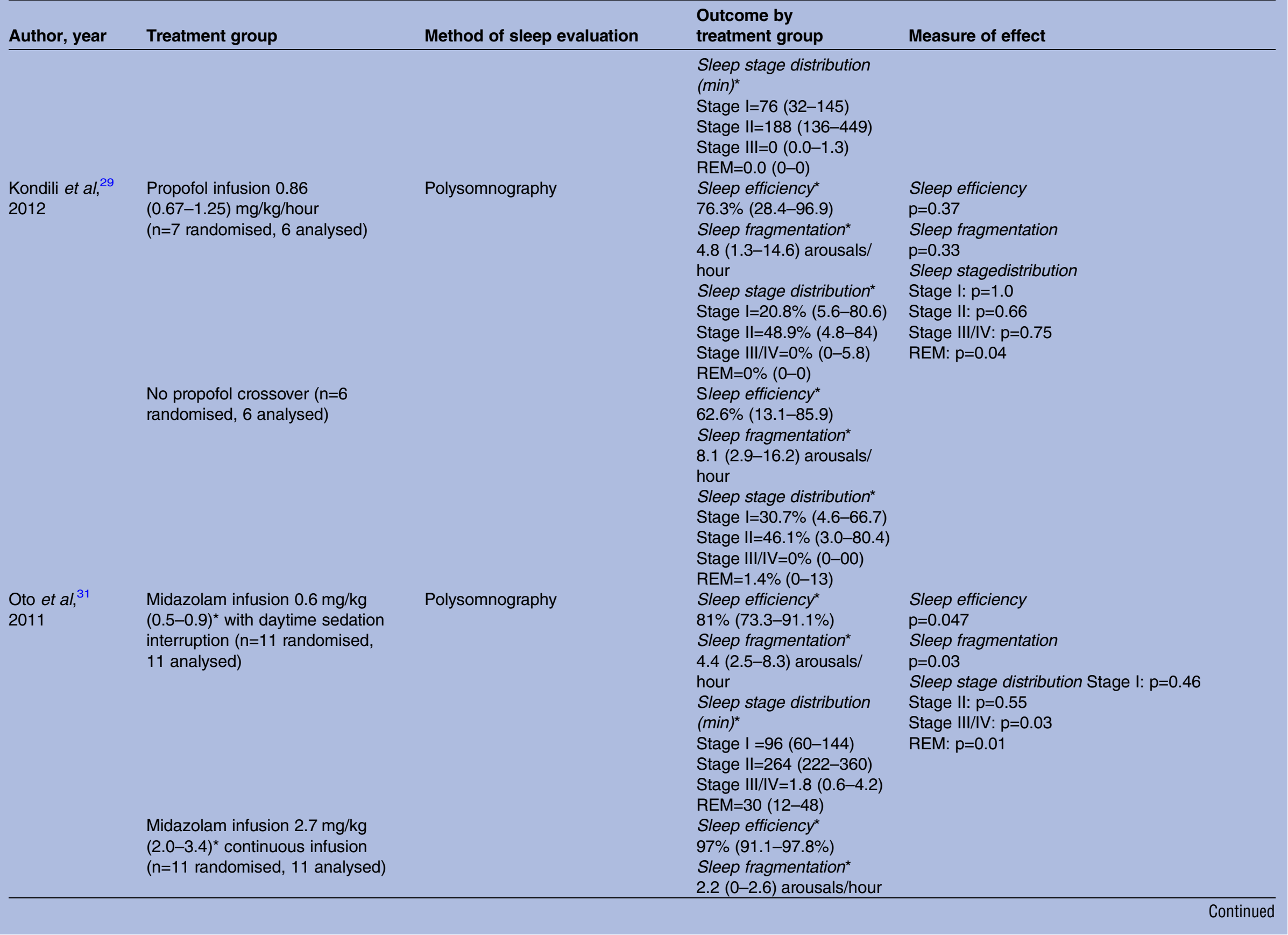




\section{Author, year Treatment group}

Melatonin $10 \mathrm{mg}$ orally $(\mathrm{n}=13$ randomised, 12 analysed) analysed)

Ibrahim et $a l^{28}$

2006

\section{Hardin et $a l^{33}$}

2006
Placebo $(\mathrm{n}=12$ randomised, 12
Melatonin 3 mg orally $(n=14$ randomised, 14 analysed)

Placebo orally ( $\mathrm{n}=18$ randomised

18 analysed)

Intermittent lorazepam iv: 0.04

$\pm 0.04 \mathrm{mg} / \mathrm{kg} /$ day $\S(\mathrm{n}=6$

randomised, 6 analysed)

\section{BIS}

Actigraphy

Nurse observation

Patient self-report questionnaire

Observation by nurse

\section{Polysomnography}

Continuous lorazepam iv

$0.72 \pm 0.39 \mathrm{mg} / \mathrm{kg} / \mathrm{day} \S(\mathrm{n}=6$

randomised, 6 analysed)

\section{Outcome by}

treatment group

Sleep stage distribution

$(\min )^{*}$

Stage I =180 (54-342)

Stage II=282 (66-468)

Stage III/IV=0 $(0-0)$

$\mathrm{REM}=0$ (0-9)

Sleep efficiency†

BIS: 39\% (27-51\%)

Actigraphy: $73 \%$

(53-93\%)

Nurse observation: $45 \% \quad$ Patient self-report: $p=0.32$

(26-64\%)

Patient self-report: $41 \%$

(24-59\%)

Sleep efficiency†

BIS: 26\% (17-36\%)

Actigraphy: $75 \%$

(67-83\%)

Nurse observation: $51 \%$

(35-68)

Patient self-report: $50 \%$

(43-58\%)

Sleep efficiency

50\% (16-69)

Sleep efficiencył

50\% (0-72\%)

Sleep efficiency§

$42 \pm 26.7 \%$

Sleep fragmentation§

$4.24 \pm 2.4$ arousals/hour

Sleep stage distribution

(\%)§

Stage $\mathrm{l}=5.8 \pm 6.6$

Stage $\|=57.7 \pm 23.8$

Stage III/IV=31.9 \pm 24.6

$\mathrm{REM}=3.6 \pm 5.7$

Sleep efficiency§

$58.8 \pm 26.3 \%$

Sleep fragmentation§

$4.79 \pm 3.7$ arousals/hour
Sleep efficiency

BIS: $p=0.09$

Actigraphy: $p=0.84$

Nurse observation: $p=0.58$

\section{Measure of effect}

Sleep efficiency

$\mathrm{p}=0.98$

Sleep efficiency

$\mathrm{p}=0.07$

Sleep fragmentation

$\mathrm{p}=0.90$

Sleep stagedistribution

Stage I: $p=0.40$

Stage II: $p=0.20$

Stage III/IV: $p=0.93$

REM: not reported 
Continuous lorazepam iv with neuromuscular blockade

$0.62 \pm 0.20 \mathrm{mg} / \mathrm{kg} / \mathrm{day} \S(\mathrm{n}=6$ randomised, 6 analysed)

Hospitalised patients not in an ICU

\section{Engelmann Propofol $2 \mathrm{mg} / \mathrm{kg} / \mathrm{hour}(\mathrm{n}=34$}

et $\mathrm{al}^{30} 2014 \quad$ randomised, 34 analysed)

Flunitrazepam bolus $0.015 \mathrm{mg} / \mathrm{kg}$

(32 randomised, 32 analysed)
Outcome by

Method of sleep evaluation

treatment group

Measure of effect

Sleep stage distribution

(\%)§

Stage $\mathrm{I}=20.8 \pm 35.5$

Stage $\|=28.34 \pm 35$

Stage III/IV $=49.6 \pm 49$

REM=NA

Sleep efficiency§

$78.8 \pm 19.6 \%$

Sleep fragmentation§

$4.4 \pm 3.7$ arousals/hour

Sleep stage distribution

(\%)§

Stage $\mathrm{l}=7.4 \pm 5.9$

Stage $\|=48.8 \pm 22.3$

Stage III/IV $=38.9 \pm 28.1$

$\mathrm{REM}=\mathrm{NA}$

BIS (analysis only in 31 propofol patients and 25 flunitrazepam

patients)

Sleep diary (PgShD) used to evaluate sleep quality, sleep

efficiency and sleep fragmentation Sleep quality recorded on 5-point scale

Sleep efficiency $¥$

BIS: 94.7\% (30.7-100)

Sleep diary (median):

$86 \%$

Sleep fragmentation

BIS (median): 0.6

arousals/hour

Sleep stage distribution

BIS values $=74.05$

(65.68-79.38)

Stage $A=7.4 \%(1.1-98)$

Stage $B=36.6 \%$

(5.4-82.3)

Stage C=5.0\% (0-50.7)

Sleep quality

Sleep diary (median)=2/5

Sleep efficiency

BIS: $92.7 \%$ (16.7-100)

Sleep diary (median):

$71 \%$

Sleep fragmentation

BIS (median): 1.1

Sleep stage distribution $\ddagger$

BIS values $=78.7$

(72.05-81)
Sleep efficiency

BIS: $p=0.777$

Sleep diary: $p=0.623$

Sleep fragmentation

BIS: $p=0.041$

Sleep stagedistribution

BIS values: $p=0.016$

Stage A: $p=0.044$

Stage $B: p=0.004$

Stage $C: p=0.69$

Sleep quality

Sleep diary: $p<0.001$ 


\section{Li Pi Shan \\ et $a{ }^{41} 2004$ \\ Lorazepam $0.5-1.0 \mathrm{mg}(\mathrm{n}=10$ randomised, 9 analysed)}

Zopiclone $3.75-7.5 \mathrm{mg}(\mathrm{n}=10$ randomised, 9 analysed)

\section{Morgan et $a{ }^{37} \quad$ Triazolam $0.25 \mathrm{mg}(\mathrm{n}=119$}

1997 randomised, 119 analysed)

Zolpidem $10 \mathrm{mg}(\mathrm{n}=120$ randomised, 120 analysed)
Nurse observation

Patient questionnaire (10-point scale for quality of sleep alertness in the morning)

Nurse observation (first 2 hours post dose only)

Sleep quality: patient self-report $1=$ excellent

2=good

$3=$ fair

4=poor

Conditioning in the morning

(patient self-report)

Visual analogue scale (0-100

where $0=$ very sleepy and $100=$ not

at all sleepy)

\section{Outcome by}

treatment group

Stage $A=30 \%(4.0-81.3)$

Stage $B=55.4 \%(96-90)$

Stage $\mathrm{C}=7.6 \%(0-83.7)$

Sleep quality

Sleep diary (median): $3 / 5$

Total sleep time§

$469 \pm 46.2 \mathrm{~min}$

Sleep quality

8.5 (7.5-10)

Conditioning in the

morning *

Alertness $=9(8-10)$

Tiredness $=7.5(5-10)$

Total sleep time

$443 \pm 37.8 \mathrm{~min}$

Sleep quality

8 (5-9)

Conditioning in the

morning ${ }^{*}$

Alertness $=9(6.5-10)$

Tiredness $=8(5.5-8.5)$

Sleep latency

Median: $30 \mathrm{~min}$

$<30 \mathrm{~min}=45 \%$

$<60 \mathrm{~min}=76 \%$

$<90 \mathrm{~min}=87 \%$

$<120 \mathrm{~min}=90 \%$

Sleep quality (mean \pm SD)

\section{$2.0 \pm 0.1$}

Conditioning in the

morning $($ mean $\pm \mathrm{SD})=62.8$

$\pm 2.5$

Total sleep time

$\mathrm{p}=0.09$

Sleep quality

$\mathrm{p}=0.17$

Conditioning in the morning

Alertness: $\mathrm{p}=0.60$

Tiredness: $p=0.29$

Sleep latency

Median: $25 \mathrm{~min}$

$<30 \mathrm{~min}=42 \%$

$<60 \mathrm{~min}=78 \%$

$<90 \mathrm{~min}=92 \%$

$<120 \mathrm{~min}=89 \%$

Sleep quality (mean \pm SD)

$2.0 \pm 0.1$ 
Placebo $(\mathrm{n}=118$ randomised, 118 analysed)

Feldmeier and Kapp, ${ }^{34} 1983$

Midazolam 15 mg orally $(n=19$ randomised, 19 analysed)

Observation by nurse

Oxazepam 15 mg orally $(\mathrm{n}=15$ randomised, 15 analysed)

Placebo $(n=16$ randomised 16 analysed)

Goetzke et al, ${ }^{38}$ 1983

Brotizolam $0.25 \mathrm{mg}$ orally $(\mathrm{n}=86$ randomised, 79 analysed but breakdown by group not reported)

Nurse observation

Patient questionnaire

Frequencies (\%) are

approximated from a bar graph

Triazolam $0.25 \mathrm{mg}$ orally

Outcome by

treatment group

Measure of effect

Conditioning in the

morning $($ mean $\pm S D)=58.4$

$+2.7$

Sleep latency

Median: $60 \mathrm{~min}$

$<30 \mathrm{~min}=22 \%$

$<60 \mathrm{~min}=49 \%$

$<90 \mathrm{~min}=64 \%$

$<120$ min $=72 \%$

Sleep quality (mean \pm SD)

$2.7 \pm 0.1$

Conditioning in the

morning $($ mean $\pm S D)=59.0$

$\pm 2.9$

Total sleep time§: $430 \mathrm{~min}$

Sleep latency: $30 \%$

asleep within $20 \mathrm{~min}$

$84 \%$ asleep within $40 \mathrm{~min}$

Total sleep time\$: $440 \mathrm{~min}$

Sleep latency: $16 \%$

asleep within $20 \mathrm{~min}$

$73 \%$ asleep within 40 min

Total sleep time: NR

Sleep latency: 0\% asleep within 20 min

$29 \%$ asleep within $40 \mathrm{~min}$

Sleep latency§ (<30 min):

55.5 patients $(70 \%)$

Sleep quality

Very good $=17 \%$

Good $=60 \%$,

Moderate $=18 \%$

Poor $=5 \%$

Conditioning in the

morning

Refreshed $=47 \%$

Little tired $=44 \%$

Very tired $=9 \%$

Sleep latency§

(<30 min): 55.5 patients

(70\%)

Sleep quality
Total sleep time

Reported as no significant difference between groups

Sleep latency (<20min)

$\mathrm{p}<0.05$ for midazolam and oxazepam vs placebo

Not reported 


\begin{tabular}{|c|c|c|c|c|}
\hline Author, year & Treatment group & Method of sleep evaluation & $\begin{array}{l}\text { Outcome by } \\
\text { treatment group }\end{array}$ & Measure of effect \\
\hline $\begin{array}{l}\text { Gallais et } a l^{35} \\
1983\end{array}$ & $\begin{array}{l}\text { Midazolam } 15 \text { mg orally ( } \mathrm{n}=19 \\
\text { randomised, } 18 \text { analysed) } \\
\text { Oxazepam } 50 \text { mg orally ( } \mathrm{n}=20 \\
\text { randomised, } 17 \text { analysed) } \\
\text { Placebo ( } \mathrm{n}=20 \text { randomised, } 16 \\
\text { analysed) }\end{array}$ & $\begin{array}{l}\text { Third-party observation (values } \\
\text { are approximated from a graph) }\end{array}$ & 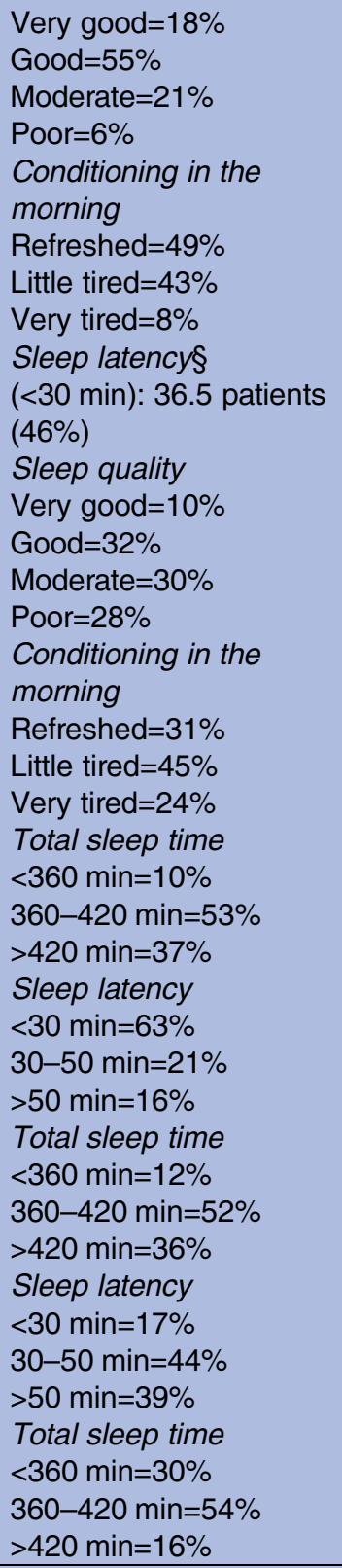 & $\begin{array}{l}\text { Total sleep time } \\
p<0.05 \text { for both drugs when compared to placebo } \\
\text { but no difference between drugs } \\
\text { Sleep latency } \\
\text { Sleep onset was shorter in the midazolam group } \\
\text { when compared with placebo }(p<0.05) \text { and } \\
\text { oxazepam ( } p, 0.05) \text {. There was no statistical } \\
\text { difference between oxazepam and placebo } \\
\text { groups }\end{array}$ \\
\hline
\end{tabular}

Very good $=18 \%$

Good $=55 \%$

Poor=6\%

Conditioning in the

ittle tired $=43 \%$

( $30 \mathrm{~min}): 36.5$ patients

Very good $=10 \%$

$\mathrm{d}=32 \%$

te $=30 \%$

morning

Refreshed $=31 \%$

Little tired $=45 \%$

Very tired $=24 \%$

$p<0.05$ for both drugs when compared to placebo

but no difference between drugs

$<360 \mathrm{~min}=12 \%$

$360-420 \mathrm{~min}=52 \%$

$<30$ min $=17 \%$

$30-50 \mathrm{~min}=44 \%$

$>50 \mathrm{~min}=39 \%$

$<360 \mathrm{~min}=30 \%$

$>420 \mathrm{~min}=16 \%$ 
Midazolam $15 \mathrm{mg}$ (40 randomised, Nurse observation

Lupolove

et $a l^{36} 1983$

40 analysed)

Oxazepam 15 mg (38 randomised, 38 analysed)
Outcome by

treatment group

Measure of effect

Sleep latency

$<30$ min $=10 \%$

$30-50 \mathrm{~min}=50 \%$

$>50 \mathrm{~min}=40 \%$

Total sleep time

$360 \mathrm{~min}=13.8 \%$

$420 \mathrm{~min}=22.5 \%$

$480 \mathrm{~min}=30.8 \%$

$540 \mathrm{~min}=14.3 \%$

$600 \mathrm{~min}=3.6 \%$

Sleep latency

$<20 \mathrm{~min}=34.4 \%$

$20-40 \mathrm{~min}=48.7 \%$

40-60 $\mathrm{min}=12.3 \%$

$>60 \mathrm{~min}=4.7 \%$

Sleep fragmentation

(arousals/night)

None $=15.9 \%$

$1-2=60.4 \%$

$>2=23.7 \%$

Total sleep time

$360 \mathrm{~min}=9.9 \%$

$420 \mathrm{~min}=41.6 \%$

$480 \mathrm{~min}=25.9 \%$

$540 \mathrm{~min}=10.9 \%$

$600 \mathrm{~min}=0.6 \%$

Sleep latency

$<20 \mathrm{~min}=15.2 \%$

$20-40 \mathrm{~min}=42.7 \%$

40-60 $\mathrm{min}=22 \%$

$>60 \mathrm{~min}=20.1 \%$

Sleep fragmentation

(arousals/night)

None $=17.5 \%$

$1-2=55.4 \%$

$>2=27.1 \%$

*Data presented as median (IQR)

†Data presented as mean $(95 \% \mathrm{Cl})$.

fData presented as median (range).

$\S$ Data presented as mean $\pm S D$.

ICU, intensive care unit; NR, not reported; PgShD, Pittsburgh Sleep Diary; REM, rapid eye movement. 


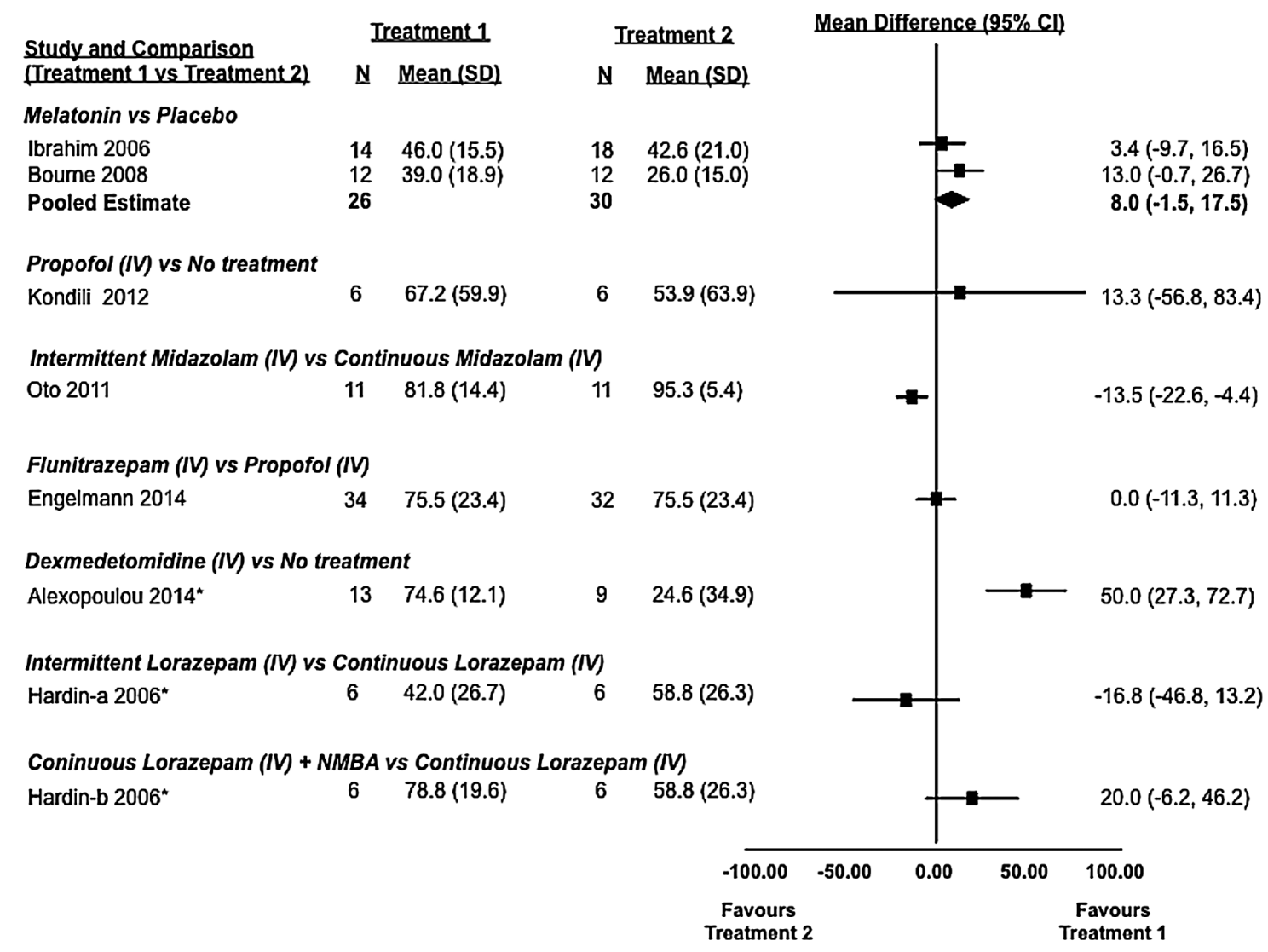

Figure 4 Sleep efficiency: observed differences from randomised and non-randomised studies and from active and inactive controlled studies (7 studies and 188 patients). Sleep efficiency was defined as the number of hours spent asleep divided by the total hours of observation. In some studies, mean and SD values were estimated using an approached described in the Methods section. *, non-randomised study; iv, intravenous; NMBA, neuromuscular blocking agent.

non-statistically significant trend in favour of melatonin when compared to placebo (mean difference of $8.0 \%$ $(95 \% \mathrm{CI}-1.5 \%$ to $17.5 \%))$.

\section{Sleep latency}

Sleep latency was evaluated in five studies. Owing to variability in assessment and reporting metrics between studies, forest plots were not created, and the appropriateness of meta-analysis was deemed low. Sleep latency data are reported for each study in table 3.

Two randomised controlled trials, Feldmeier and $\operatorname{Kapp}^{34}(\mathrm{n}=50)$ and Gallais $e t a l^{35}(\mathrm{n}=51)$, compared the effects of midazolam and oxazepam to placebo. One randomised controlled trial by Lupolover et $a l^{36}(\mathrm{n}=78)$ compared midazolam to oxazepam. Sleep latency was measured by third-party observation or patient questionnaire in all three studies. The studies by Feldmeier and Kapp and Gallais et al reported a statistically significant reduction in sleep latency with midazolam $(15 \mathrm{mg}$ orally) compared to placebo (median difference of $30-53 \%, \mathrm{p}<0.05)$; however, only Feldmeier and Kapp ${ }^{34}$ found oxazepam ( $15 \mathrm{mg}$ orally) to be superior to placebo (median difference $16 \%, \mathrm{p}<0.05$ ). Gallais $e t a l^{35}$ found midazolam $15 \mathrm{mg}$ orally to be superior to oxazepam $50 \mathrm{mg}$ orally (table 3) as did Lupolover et $a l^{36}$
(15 mg doses for midazolam and oxazepam), but only after 3 days of treatment (table 3 ).

Two randomised control trials evaluated sleep latency with triazolam. ${ }^{37}{ }^{38}$ Morgan et al $(\mathrm{n}=357)$ compared triazolam to zolpidem and placebo, while Goetzke et al $(\mathrm{n}=79)$ compared it to brotizolam and placebo. In both cases, drug therapy (any) appeared to be superior to placebo with respect to sleep latency; however, no statistical analysis was provided by Goetzke $e t a l .{ }^{38}$ No statistically significant differences were identified between the active arms of either study (table 3).

\section{Sleep fragmentation}

Sleep fragmentation was evaluated in five studies: three randomised controlled trials ${ }^{29-31}$ and two cohort studies $^{32} 33$ (table 3, figure 5). Variability in treatments studied precluded the opportunity for meta-analysis. Two randomised controlled trials studied the effect of propofol on the number of awakenings compared to flunitrazepam $^{30}$ or no treatment. $^{29}$ Engelmann et $a l^{30}$ $(\mathrm{n}=66)$, using BIS, found that propofol (median: 0.6 arousals per hour of sleep) was associated with statistically significantly fewer sleep interruptions than flunitrazepam (median: 1.1 arousals per hour of sleep in critically ill patients, $\mathrm{p}=0.041$ ). However, Kondili et $a l^{29}$ 


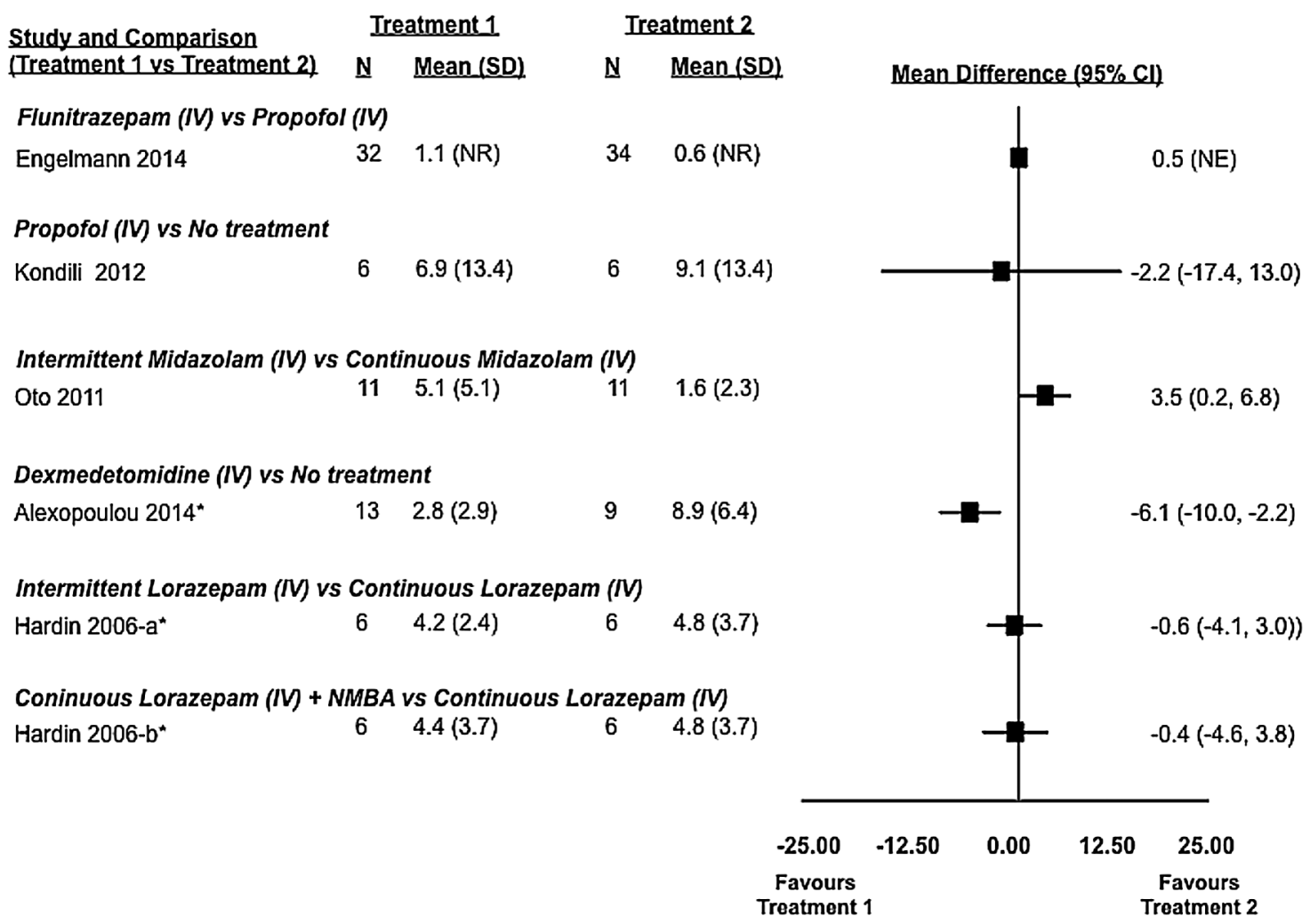

Figure 5 Sleep fragmentation: observed differences from randomised and non-randomised studies and from active and inactive controlled studies (5 studies and 125 patients). Sleep fragmentation was defined as the number of arousals or awakenings per hour of sleep. In some studies, mean and SD values were estimated using an approached described in the Methods section. *, non-randomised study; iv, intravenous; NE, not estimable; NMBA, neuromuscular blocking agent; NR, not reported.

$(n=12)$, using polysomnography, found insufficient evidence of a difference between patients receiving propofol (median (IQR) 4.8 (1.3-14.6) awakenings per hour of sleep) and those receiving no treatment (median (IQR) 8.1 (2.9-16.2) awakenings per hour of sleep).

Dexmedetomidine compared to no treatment was found to statistically significantly reduce the number of sleep interruptions in critically ill patients. Using polysomnography, Alexopoulou et $a l^{32}(\mathrm{n}=23)$ found that patients receiving infusions of dexmedetomidine had a median of 2.2 (IQR 1.6-4.5) awakenings per hour, while those receiving no treatment had a median of 7.1 (IQR 1.6-4.5) awakenings per hour ( $\mathrm{p}=0.0023)$.

Two trials comparing different dosing strategies for benzodiazepines in critically ill patients yielded conflicting results. Oto $e t a \hat{l}^{1}$ found that continuous infusions of midazolam were associated with fewer awakenings when compared to infusions incorporating daytime interruptions, while Hardin $e t a l^{33}$ found no difference between continuous infusions of lorazepam (with and without a neuromuscular blocker) and an intermittent dosing strategy.

\section{Sleep stage distribution}

Two randomised control trials, ${ }^{29}{ }^{31}$ two cohort studies $^{32} 33$ and two case series ${ }^{39} 40$ evaluated sleep architecture using polysomnography in a total of 74 patients.
Treatment effects from comparative studies are shown in figure 6 , while data for all studies are found in table 3 . Irrespective of treatment allocation, patients in hospital primarily experience light non-restorative sleep, corresponding to stages N1 and N2. Only Hardin et al (continuous infusion of lorazepam with or without neuromuscular blocker vs interrupted infusion) reported a proportion of sleep as stage N3 (restorative sleep), where stage N3 represented $32-50 \%$ of total sleep but insufficient evidence of a difference between groups. No other trial using polysomnography was able to describe meaningful amounts of stage N3 sleep. Rapid eye movement (REM) sleep was only described with midazolam infusions interrupted during the day $(6.8 \%)$, continuous infusions of midazolam (2\%), interrupted lorazepam infusions $(3.6 \%)$ and the no treatment arm of the study by Kondili et al (1.4\%). ${ }^{29} 313339$ Given the variability in studied treatments, study designs and the limited number of overall studies, meta-analysis was not attempted.

\section{Secondary outcomes}

Delirium was not assessed in the included studies; however, Ibrahim et $a l^{28}$ evaluated agitation using the Riker Sedation-Agitation Scale and found no difference between melatonin $(n=14)$ and placebo $(n=18)$ groups. Cognitive function was evaluated in the study by $\mathrm{Li} \mathrm{Pi}$ 


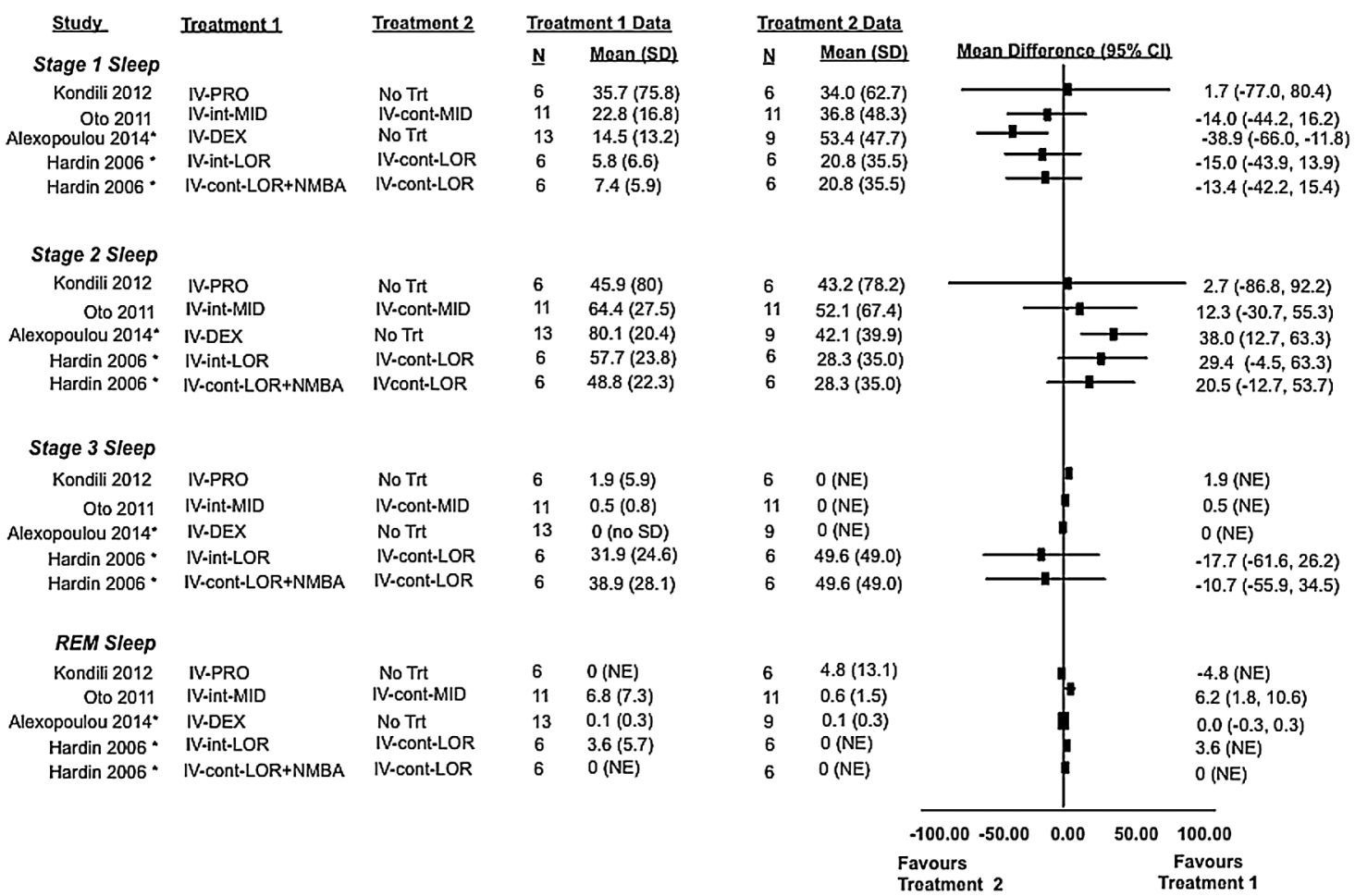

Figure 6 Sleep architecture: observed differences from randomised and non-randomised studies and from active and inactive controlled studies (4 studies and 59 patients). Sleep stage distribution defined as percentage of total sleep time patients spent in each stage. In some studies, mean and SD values were estimated using an approached described in the Methods section. *, non-randomised study; cont, continuous dosing; DEX, dexmedatomidine; int, intermittent dosing; iv, intravenous; LOR, Iorazepam; MID, midazolam; NE, not estimable; NMBA, neuromuscular blocking agent; No Trt, no treatment; PRO, propafol.

Shan and Ashworth ${ }^{41}$ using the mini mental state examination, and no difference was found between lorazepam $(n=9)$ and zopiclone $(n=9)$. Narcotic requirements were evaluated in the study by Oto $e t a l .^{31}$ While more patients $(9 / 11=81.8 \%)$ in the group assigned to midazolam infusions with daily interruption required opioids than those assigned to the continuous infusion group $(4 / 11=36.4 \%)$, there was no difference in median dose between groups. In the study by Hardin $e t a l^{33}(\mathrm{n}=18)$, patients assigned to continuous infusions of lorazepam $(n=6)$ required significantly more morphine $(0.48 \pm 0.52 \mathrm{mg} / \mathrm{kg} /$ day $)$ than those assigned to interrupted infusions $(0.11 \pm 0.13 \mathrm{mg} / \mathrm{kg} /$ day $)$ $(n=6)$ or continuous infusions with neuromuscular blockade $(0.14 \pm 0.32 \mathrm{mg} / \mathrm{kg} /$ day $) \quad(\mathrm{n}=6) \quad(\mathrm{p}=0.031)$. No other differences were found between groups.

Duration of mechanical ventilation, length of stay, occurrence of infection, glucose control, hypertension, myocardial infarction and mortality were not described in any included study.

\section{Safety}

No study reported occurrence of serious life-threatening events. Seven of the 15 studies did not report any safety information in the methods or results. Of the remaining eight, ${ }^{27} 3034-3841$ no study reported any difference with respect to adverse events between the active treatment groups compared to the placebo or no treatment arms.
Engelmann $e t a l^{30}$ reported that three patients developed respiratory depression after flunitrazepam administration requiring the insertion of a nasopharyngeal airway but not withdrawal from the study. One patient receiving dexmedetomidine in the study by Alexopoulou $e t a \vec{l}^{2}$ had the study drug discontinued for bradycardia (heart rate $<50 \mathrm{bpm}$ ).

Patient conditioning, defined as somnolence or alertness the morning following administration of hypnotics, was reported in five studies. Four reported no differences between drugs and/or placebo. ${ }^{34} 373841$ The study by Lupolover $e t a l^{36}$ described an improved state on awakening by patient self-report in those taking midazolam compared to oxazepam.

Headache and nausea were the most commonly reported adverse events. Discontinuation of therapy due to nausea was only observed in the placebo group of Morgan $e t$ al. $^{37}$ Allergic type skin reactions were reported in two patients-one was associated with triazolam; the other, described as pruritus, occurred in a patient with pancreatitis and whether the reaction was attributed to benzodiazepines or placebo was not specified in the study. ${ }^{36} 38$

\section{DISCUSSION}

In this review, we set out to summarise the existing evidence and compare studies looking at the efficacy and 
safety of various pharmacological agents for sleep. Fifteen studies encompassing 861 patients met our inclusion criteria; however, clinical and methodological heterogeneity and variable outcome reporting were judged to preclude performance of reliable meta-analyses in all cases except for two trials comparing melatonin to placebo with similar methods for evaluating sleep efficiency. An apparent reduction in sleep latency with pharmacotherapy when compared to placebo or no treatment has been demonstrated although not consistently across all studies evaluating this metric. Sleep efficiency and sleep fragmentation were not shown to be improved by pharmacological therapy when compared to placebo or no treatment. This is in keeping with expert opinion that pharmacological agents, while somewhat effective for sleep onset insomnia, are ineffective for maintaining sleep. ${ }^{42}$

Furthermore, sleep architecture in hospital, as measured by polysomnography, is dominated by stage N1 and N2 sleep. Restorative N3 sleep or REM sleep was found to be rare regardless of pharmacological intervention. When sleep is highly fragmented, it is common to observe a predominance of N1 sleep, as an arousal in an epoch of sleep on the EEG results in the sleep stage being scored back to stage N1. ${ }^{43}$ Stage N3 sleep was observed only in a single study, in patients receiving continuous or intermittently administered lorazepam. ${ }^{33}$ A paucity of N3 sleep is not unusual in middle-aged or elderly populations, where N3 typically constitutes only $5-10 \%$ of sleep. ${ }^{44}$ Furthermore, the sleep stages are influenced by the medications studied. Benzodiazepines, for example, are potent suppressors of N3 sleep, and increase the presence of sleep spindles. ${ }^{45}$ Spindles are the hallmark of N2 sleep, so it is common to see increased N2 sleep and decreased N3 sleep with benzodiazepines. Concurrent opioids will also decrease N3 sleep. ${ }^{46}$ The large percentage of N3 sleep seen in the study in which lorazepam was used is surprising given the intervention is known to suppress N3 sleep. ${ }^{33}$ Furthermore, this finding is not replicated in any other studies. A possible explanation is that N3 sleep is commonly overscored. REM sleep was only observed in four studies, independent of drug or placebo allocation. REM sleep can also be influenced by current and prior medications. There is evidence to suggest that benzodiazepines decrease REM sleep. ${ }^{47}$ Sleep stage distribution is also influenced by prior sleep episodes and medications. For example, recent sleep deprivation will increase N3 sleep and REM sleep. ${ }^{48}$ Lastly, recent medication use prior to the polysomnography recording may influence sleep stages (ie, recent discontinuation of REM-suppressant, such as a barbiturate, antidepressant or alcohol, will result in REM rebound). In summary, the disappointing performance of the sedatives in effecting greater amounts of N3 and REM is in part due to the well-known effect of these drugs on sleep stages. In addition, the medications administered and discontinued, as well as prior sleep during the run-in period, can influence sleep architecture, and although some of the trials were randomised, it is possible that these factors could be confounding the results given the small sample sizes. It is also important to recognise that while medication may influence the quality of sleep in hospital, severity of illness and environmental factors are important confounders. Severity of illness among patients included in this review ranged from critical illness requiring mechanical ventilation to admissions for elective surgery. Severity of illness was not described in some studies. Although our study selection strategy was designed to identify acutely ill adults, their variability in severity of illness within this group is still large. Furthermore, most studies did not describe the presence, or evaluate the impact, of environmental factors such as noise, light and interruptions to provide care that contribute to insomnia in hospital. Attempts to minimise or standardise the influence of environmental factors (ie, use of earplugs or eye covers, 'quiet times' in the unit) were not documented or, perhaps, attempted. Given the results of our review and others of non-pharmacological interventions, a multimodal approach that involves minimisation of environmental factors, pharmacotherapy and treatment of the underlying disease would be worthy of future study.

The lack of high-quality randomised controlled trials is one of the limitations of this review. The diversity of outcome reporting and the high degree of methodological heterogeneity prevented statistical pooling of the results in almost all circumstances. Major sources of clinical heterogeneity included large variability in drug dosing (ie, oxazepam dosing ranged from 15 to $50 \mathrm{mg}$ per dose), severity of illness in hospital and the method by which sleep was evaluated. Only 6 of the 15 studies included used polysomnography which is considered the gold standard for sleep evaluation. Only two of these were randomised controlled trials, which speaks to the practical difficulty in using polysomnography in large trials and sick patients. In fact, 28 potentially eligible trials were excluded at second-level screening because the sole measure of sleep outcomes relied on patient self-reporting. We elected to exclude these studies as self-reporting has been demonstrated to correlate poorly with objective assessments and is associated with systematic bias. ${ }^{14}$ Furthermore, the general lack of safety outcome reporting does not allow a thorough evaluation of risk. Given paucity of convincing efficacy data, the relatively high potential for adverse events with these classes of drugs and the vulnerability of the population being studied future studies must systematically evaluate risk in order for clinicians to adequately assess the risk/ benefit relationship of these interventions. Language restriction to English and French may also be considered a limitation although others have shown that this restriction may have little to no impact on findings. ${ }^{16}$

It is also important to recognise the paucity of placebo-controlled trials in this setting. An evidencebased approach to clinical trial design would suggest 
that placebo-controlled trials that evaluate the efficacy of drug therapies be conducted in advance of comparative trials. While placebo-controlled trials are available in healthy volunteers and otherwise healthy communitybased patients, the aetiology of sleep disturbances is not the same as that of the acutely ill patient sleeping in a hospital bed. Similarly, the outcomes of such trials cannot be extrapolated to this population either. Given that the majority of hospitalised patients receive pharmacological sleep aids in hospital in the absence of evidence to suggest that they are helpful and safe, well-designed trials are needed. Future studies must be randomised, employ a placebo arm, use standardised definitions of sleep-related outcomes and evaluate sleep using objective measures such as polysomnography.

\section{CONCLUSIONS}

While sleep is universally recognised as an essential body function to enable the restoration of physiological systems, sleep disturbances are common in hospitalised patients. A variety of pharmacological agents are used in the majority of these patients with the presumption that they improve the quality and quantity of sleep; however, this review suggests that the low-to-moderate quality evidence available does not support this notion. While drug therapy may be associated with falling asleep faster (although not consistently demonstrated), there is no evidence that drug therapy improves the more important outcomes of sleep efficiency or quality. This may be due to the fact that drug therapy is truly ineffective or that the quality of existing trials is inadequate to show a difference. It is evident that larger, better quality trials are needed to definitively answer this question. Future trials must use standardised definitions for sleep-related outcomes, objectives methods for measurement, a placebo arm and systematically evaluate risk. At this time, there is insufficient evidence to support the current usage of pharmacotherapy for the treatment of sleep disturbances among hospitalised adults.

\author{
Author affiliations \\ ${ }^{1}$ Department of Pharmacy and Critical Care, The Ottawa Hospital, Ottawa, \\ Ontario, Canada \\ ${ }^{2}$ The Ottawa Hospital Research Institute, Ottawa, Ontario, Canada \\ ${ }^{3}$ Department of Pharmacy, Hôpital Montfort-The Ottawa Hospital, Ottawa, \\ Ontario, Canada \\ ${ }^{4}$ School of Epidemiology, Public Health and Preventative Medicine, University \\ of Ottawa, Ottawa, Ontario, Canada \\ ${ }^{5}$ Department of Pharmacy, Mount Sinai Hospital, University of Toronto, \\ Toronto, Ontario, Canada \\ ${ }^{6}$ Department of Critical Care, The Ottawa Hospital, Ottawa, Ontario, Canada \\ ${ }^{7}$ Department of Pharmacy, The Ottawa Hospital, Ottawa, Ontario, Canada \\ ${ }^{8}$ Department of Respirology, Thunder Bay Regional Health Sciences Centre, \\ Northern Ontario School of Medicine, Sudbury, Ontario, Canada
}

Acknowledgements The authors acknowledge Risa Shorr MLS, an information specialist, for her assistance in developing and validating our search strategy. The authors also acknowledge Dr Dean Fergusson for reviewing the manuscript.
Contributors All authors (SK, AM, BH, LB, ER, EM and VL) contributed to the study protocol. SK and AM were responsible for abstract screening, study selection and data extraction. BH, SK and AM performed the analysis and all authors contributed to the interpretation of results and writing of the final manuscript.

Funding This research received no specific grant from any funding agency in the public, commercial or not-for-profit sectors.

\section{Competing interests None declared.}

Provenance and peer review Not commissioned; externally peer reviewed.

Data sharing statement All data were extracted from previously published papers which are referenced in the manuscript.

Open Access This is an Open Access article distributed in accordance with the Creative Commons Attribution Non Commercial (CC BY-NC 4.0) license, which permits others to distribute, remix, adapt, build upon this work noncommercially, and license their derivative works on different terms, provided the original work is properly cited and the use is non-commercial. See: http:// creativecommons.org/licenses/by-nc/4.0/

\section{REFERENCES}

1. Young JS, Bourgeois JA, Hilty DM, et al. Sleep in hospitalized medical patients, part 1: factors affecting sleep. J Hosp Med 2008;3:473-82.

2. Pisani MA, Friese RS, Gehlbach BK, et al. Sleep in the intensive care unit. Am J Respir Crit Care Med 2015;191:731-8.

3. Bano M, Chiaromanni F, Corrias M, et al. The influence of environmental factors on sleep quality in hospitalized medical patients. Front Neurol 2014;5:267.

4. Isaia G, Corsinovi L, Bo M, et al. Insomnia among hospitalized elderly patients: prevalence, clinical characteristics and risk factors Arch Gerontol Geriatr 2011;52:133-7.

5. Weinhouse GL, Schwab RJ, Watson PL, et al. Bench-to-bedside review: delirium in ICU patients-importance of sleep deprivation. Crit Care 2009;13:234.

6. Kamdar BB, Niessen T, Colantuoni E, et al. Delirium transitions in the medical ICU: exploring the role of sleep quality and other factors. Crit Care Med 2015;43:135-41.

7. Raymond I, Ancoli-Israel S, Choiniere M. Sleep disturbances, pain and analgesia in adults hospitalized for burn injuries. Sleep Med 2004;5:551-9.

8. Manabe K, Matsui T, Yamaya M, et al. Sleep patterns and mortality among elderly patients in a geriatric hospital. Gerontology 2000;46: 318-22.

9. Morin CM, Jarrin DC. Epidemiology of insomnia: prevalence, course, risk factors, and public health burden. Sleep Med Clin 2013;8:281-97.

10. Young JS, Bourgeois JA, Hilty DM, et al. Sleep in hospitalized medical patients, part 2: behavioral and pharmacological management of sleep disturbances. J Hosp Med 2009;4:50-9.

11. Tamrat R, Huynh-Le MP, Goyal M. Non-pharmacologic interventions to improve the sleep of hospitalized patients: a systematic review. J Gen Intern Med 2014;29:788-95.

12. Flaherty $\mathrm{JH}$. Insomnia among hospitalized older persons. Clin Geriatr Med 2008;24:51-67:vi.

13. Richardson WS, Wilson MC, Nishikawa J, et al. The well-built clinical question: a key to evidence-based decisions. ACP J Club 1995;123:A12-13.

14. Lauderdale DS, Knutson KL, Yan LL, et al. Self-reported and measured sleep duration: how similar are they? Epidemiology 2008;19:838-45.

15. Cespedes EM, Hu FB, Redline S, et al. Comparison of self-reported sleep duration with actigraphy: results from the hispanic community health study/study of latinos sueno ancillary study. Am J Epidemiol 2016;183:561-73.

16. Morrison A, Polisena J, Husereau D, et al. The effect of Englishlanguage restriction on systematic review-based meta-analyses: a systematic review of empirical studies. Int $J$ Technol Assess Health Care 2012;28:138-44.

17. Sampson M, McGowan J, Cogo E, et al. An evidence-based practice guideline for the peer review of electronic search strategies. J Clin Epidemiol 2009;62:944-52.

18. Balshem $\mathrm{H}$, Stevens $\mathrm{A}$, Ansari $\mathrm{M}$, et al. Finding grey literature evidence and assessing for outcome and analysis reporting biases when comparing medical interventions: $A H R Q$ and the effective health care program. Methods guide for effectiveness and 
comparative effectiveness reviews. (Prepared by the Oregon Health and Science University and the University of Ottawa Evidence-based Practice Centers under Contract Nos. 290-200710057-I and 290-2007-10059-I.) AHRQ Publication

No. 13(14)-EHC096-EF. Rockville, MD: Agency for Healthcare Research and Quality. November 2013. www.effectivehealthcare. ahrq.gov/reports/final.cfm

19. Moher D, Liberati A, Tetzlaff J, et al. Preferred reporting items for systematic reviews and meta-analyses: the PRISMA statement. PLoS Med 2009;6:e1000097.

20. Hozo SP, Djulbegovic B, Hozo I. Estimating the mean and variance from the median, range, and the size of a sample. BMC Med Res Methodol 2005;5:13.

21. Wan X, Wang W, Liu J, et al. Estimating the sample mean and standard deviation from the sample size, median, range and/or interquartile range. BMC Med Res Methodol 2014;14:135.

22. Higgins JP, Altman DG, Gotzsche PC, et al. The Cochrane Collaboration's tool for assessing risk of bias in randomised trials. BMJ 2011;343:d5928.

23. The Newcastle-Ottawa Scale (NOS) for assessing the quality of nonrandomised studies in meta-analyses. 2013. http://www.ohri.ca/ programs/clinical_epidemiology/oxford.asp (accessed 28 Jan 2015).

24. Higgins JP, Thompson SG. Quantifying heterogeneity in a meta-analysis. Stat Med 2002;21:1539-58.

25. Raudenbush SW. Analyzing effect sizes: random effects models. New York: Russell Sage Foundation, 2009.

26. Viechtbauer W. Bias and efficiency of meta-analytic variance estimators in the random-effects model. J Educ Behav Stat 2005;30:261-93.

27. Bourne RS, Mills GH, Minelli C. Melatonin therapy to improve nocturnal sleep in critically ill patients: encouraging results from a small randomised controlled trial. Crit Care 2008;12:R52.

28. Ibrahim MG, Bellomo R, Hart GK, et al. A double-blind placebo-controlled randomised pilot study of nocturnal melatonin in tracheostomised patients. Crit Care Resusc 2006;8:187-91.

29. Kondili E, Alexopoulou C, Xirouchaki N, et al. Effects of propofol on sleep quality in mechanically ventilated critically ill patients: a physiological study. Intensive Care Med 2012;38:1640-6.

30. Engelmann C, Wallenborn J, Olthoff D, et al. Propofol versus flunitrazepam for inducing and maintaining sleep in postoperative ICU patients. Indian J Crit Care Med 2014;18:212-19.

31. Oto J, Yamamoto K, Koike S, et al. Effect of daily sedative interruption on sleep stages of mechanically ventilated patients receiving midazolam by infusion. Anaesth Intensive Care 2011;39:392-400

32. Alexopoulou C, Kondili E, Diamantaki E, et al. Effects of dexmedetomidine on sleep quality in critically ill patients: a pilot study. Anesthesiology 2014;121:801-7.

33. Hardin KA, Seyal M, Stewart T, et al. Sleep in critically ill chemically paralyzed patients requiring mechanical ventilation. Chest 2006;129:1468-77.
34. Feldmeier C, Kapp W. Comparative clinical studies with midazolam, oxazepam and placebo. Br J Clin Pharmacol 1983;16(Suppl 1): 151S-5S.

35. Gallais $\mathrm{H}$, Casanova $\mathrm{P}$, Fabregat $\mathrm{H}$. Midazolam and oxazepam in the treatment of insomnia in hospitalized patients. $\mathrm{Br} J \mathrm{Clin}$ Pharmacol 1983;16(Suppl 1):145S-9S.

36. Lupolover R, Ballmer U, Helcl J, et al. Efficacy and safety of midazolam and oxazepam in insomniacs. Br J Clin Pharmacol 1983;16(Suppl 1):139S-43S.

37. Morgan PJ, Chapados R, Chung FF, et al. Evaluation of zolpidem, triazolam, and placebo as hypnotic drugs the night before surgery. J Clin Anesth 1997;9:97-102.

38. Goetzke E, Findeisen P, Welbers IB. Comparative study on the efficacy of and the tolerance to the triazolodiazepines, triazolam and brotizolam. Br J Clin Pharmacol 1983;16(Suppl 2):407S-12S.

39. Kim SJ, Park J, Lee YJ, et al. The optimal dose of midazolam for promoting sleep in critically III patients: a pilot study. Korean $J$ Crit Care Med 2014;29:166-71.

40. Oto J, Yamamoto K, Koike S, et al. Sleep quality of mechanically ventilated patients sedated with dexmedetomidine. Intensive Care Med 2012;38:1982-9.

41. Li Pi Shan RS, Ashworth NL. Comparison of lorazepam and zopiclone for insomnia in patients with stroke and brain injury: a randomized, crossover, double-blinded trial. Am J Phys Med Rehabil 2004;83:421-7.

42. Rosenberg RP. Sleep maintenance insomnia: strengths and weaknesses of current pharmacologic therapies. Ann Clin Psychiatry 2006;18:49-56.

43. Berry RB, Budhiraja R, Gottlieb DJ, et al, American Academy of Sleep Medicine. Rules for scoring respiratory events in sleep: update of the 2007 AASM Manual for the Scoring of Sleep and Associated Events. Deliberations of the Sleep Apnea Definitions Task Force of the American Academy of Sleep Medicine. J Clin Sleep Med 2012;8:597-619.

44. Achermann P, Borbély AA. Chapter 37-sleep homeostasis and models of sleep regulation. In: Dement MHKRC,ed. Principles and practice of sleep medicine. 5th edn. Philadelphia: W.B. Saunders, 2011:431-44

45. Poyares D, Guilleminault C, Ohayon MM, et al. Chronic benzodiazepine usage and withdrawal in insomnia patients. J Psychiatr Res 2004;38:327-34

46. Dimsdale JE, Norman D, DeJardin D, et al. The effect of opioids on sleep architecture. J Clin Sleep Med 2007;3:33-6.

47. Borbely AA, Mattmann P, Loepfe M, et al. Effect of benzodiazepine hypnotics on all-night sleep EEG spectra. Hum Neurobiol 1985;4:189-94.

48. Brunner DP, Dijk DJ, Tobler I, et al. Effect of partial sleep deprivation on sleep stages and EEG power spectra: evidence for non-REM and REM sleep homeostasis. Electroencephalogr Clin Neurophysiol 1990;75:492-9. 